\title{
Bounded and Integrable Automorphic Forms
}

\section{MARVIN ISADORE KNOPP}

\author{
Communicated by the Editors
}

1. Introduction. In the past few years there has been a great deal of interest in the Bers spaces $A_{\alpha, \mu}(G)$ and $B_{\alpha, \mu}(G)$, of bounded and integrable automorphic forms, respectively on the unit disc $U$ [1]. (Definitions are given below.) In particular it is still an open question whether

$$
A_{\alpha, \mu}(G) \subset B_{\alpha, \mu}(G)
$$

holds for $q>1$ and an arbitrary discrete group $G$ of linear fractional transformations acting on $U$. Several years ago Drasin and Earle [2] showed that (1) holds if $G$ is finitely generated, $q$ is an integer $\geqq 2$, and the multiplier system $\mu$ is identically 1 . Recently Metzger and Rao [7] gave a necessary and sufficient condition for (1) to hold with arbitrary discrete $G$ and $q>1$, and made use of this to prove (1) for finitely generated $G$ without parabolic elements, but with arbitrary $q>1$ and arbitrary multiplier system $\mu$. More recently still, the latter two authors [8] have applied a result of [5] to derive (1) for any finitely generated $G$ (with or without parabolic elements), and arbitrary $q>1$ and multiplier system $\mu$. [8] also applies the method of [2] to give a proof that

$$
A_{1, \mu}(G)=\{0\}
$$

for finitely generated $G$ of the second kind. (For finitely generated $G$ of the first kind (2) may or may not hold, depending on $G$ and $\mu$.)

The purpose of this note is to prove (1) in the same generality as do Metzger and Rao, but by a generalization of the original method of [2], and to observe that this same method yields

$$
A_{\alpha, \mu}(G)=\{0\}, \quad \text { if } q \leqq 1,
$$

for finitely generated $G$ of the second kind and arbitrary multiplier system $\mu$. We summarize the results in the following

Theorem. Suppose $G$ is finitely generated of the second kind, $q$ is an arbitrary 
real number, and $\mu$ is a multiplier system connected with the degree $-2 q$ and the group $G$.

(a) If $q>1$, then $A_{\alpha, \mu}(G) \subset B_{\alpha, \mu}(G)$.

(b) If $q \leqq 1$, then $A_{\alpha, \mu}(G)=\{0\}$.

The principal tool in the proof is a generalization of the lemma of [2]. This generalization is stated in $\$ 2$.

2. Definitions and the statement of Lemma 1. The functions in question here satisfy the functional equations,

$$
f(V z)=\mu(V) V^{\prime}(z)^{-q} f(z), \quad z \varepsilon U, \quad V \varepsilon G,
$$

where $q$ is a real number, $\mu(V)$ is independent of $z$, and $|\mu(V)|=1$ for each $V \varepsilon G$. If $f$ satisfies (4) and is meromorphic in $U$, we call $f$ an automorphic form of degree $-2 q$ on $G$, with multiplier system $\mu$. If $f \neq \equiv 0$ on $U$ it follows from (4) that $\mu$ satisfies the consistency condition

$$
\mu\left(V_{1} V_{2}\right)\left(V_{1} V_{2}\right)^{\prime}(z)^{-a}=\mu\left(V_{1}\right) \mu\left(V_{2}\right) V_{1}^{\prime}\left(V_{2} z\right)^{-a} V_{2}^{\prime}(z)^{-q},
$$

for $V_{1}, V_{2} \varepsilon G$. If $\mu$ satisfies (5) and $|\mu(V)|=1$ for all $V \varepsilon G$, then $\mu$ is called a multiplier system for the group $G$ and the degree $-2 q$, whether or not $\mu$ occurs in a functional equation of the form (4).

Let $\lambda(z)=\left(1-|z|^{2}\right)^{-1}, z \varepsilon U$, the Poincaré metric for $U$. The Bers space $A_{a, \mu}(G)$ of integrable automorphic forms consists of those functions $f$ satisfying (4) which are holomorphic in $U$ and such that

$$
\|f\|_{A, G}=\iint_{\Omega} \lambda(z)^{2-a}|f(z)| d x d y<\infty,
$$

where $R$ is a measurable fundamental domain for $G$ whose boundary has Lebesgue measure 0 . The space $B_{a, \mu}(G)$ of bounded automorphic forms is the set of those $f$ holomorphic in $U$ which satisfy (4) and such that

$$
\|f\|_{B, G}=\sup _{z \varepsilon \Omega} \lambda(z)^{-a}|f(z)|<\infty .
$$

It is easy to verify that $A_{\alpha, \mu}(G)$ and $B_{q, \mu}(G)$ are Banach spaces under the norms $\|f\|_{A}$ and $\|f\|_{B}$, respectively. There is a third Banach space, introduced in [5], which will be useful. We say $f$ is in $H_{a, \mu}(G)$ if $f$ is holomorphic in $U$, satisfies (4), and $f$ is bounded on $R$, i.e., $f \varepsilon H^{\infty}(R)$. The norm in $H_{\alpha, \mu}(G)$ is then given by

$$
\|f\|_{H, G}=\sup _{z \varepsilon \Omega}|f(z)| \text {. }
$$

In the case $G=\{i d\}$, then $R=U$ and the three Banach spaces of automorphic forms are denoted $A_{q}, B_{q}$, and $H_{q}\left(=H^{\infty}\right)$. It is not hard to show that (see [5])

$$
H_{a, \mu}(G) \subset A_{a, \mu}(G), \quad \text { if } q>1 \text {, }
$$

and

$$
H_{a, \mu}(G) \subset B_{a, \mu}(G), \quad \text { if } \quad q \geqq 0 \text {. }
$$


Lemma 1. (a) If $q>1$, then there exists $F \varepsilon H_{\alpha, \mu}(G)$ such that $\Psi / F \varepsilon A_{a}$ for all $\Psi \varepsilon A_{a, \mu}(G)$.

(b) If $q \leqq 1$, then there exists $F$ holomorphic in $U$, satisfying (4), and such that $\Psi / F$ \& $A_{1}$ for all $\Psi \varepsilon A_{\alpha, \mu}(G)$.

The proof of Lemma 1 will be given in $\$ 4$.

3. Proof of the theorem. We briefly indicate the argument of Drasin and Earle [2], which shows that the theorem follows from Lemma 1. Suppose first that $q>1$ and $\Psi \varepsilon A_{\alpha, \mu}(G)$. By part (a) of Lemma 1, there exists $F \varepsilon H_{q, \mu}(G)$ such that $\Psi / F=f \varepsilon A_{q}$. To prove that $\Psi \varepsilon B_{q, \mu}(G)$, consider

$$
\begin{aligned}
\|\Psi\|_{B, G}=\|F \cdot f\|_{B, G}=\sup _{z \varepsilon Q} \lambda(z)^{-a} \mid F(z) & \| f(z) \mid \\
& \leqq\|F\|_{H, G} \cdot\|f\|_{B, G} \leqq\|F\|_{H, G} \cdot\|f\|_{B,(i d)},
\end{aligned}
$$

where, of course,

$$
\|f\|_{B,(i d)}=\sup _{z \varepsilon U} \lambda(z)^{-a}|f(z)| .
$$

But Bers [1, p. 199] has shown that $A_{a} \subset B_{q}$, so that $\|f\|_{B,(i d)}<\infty$, since $f \varepsilon A_{a} .{ }^{1}$ On the other hand, $\|F\|_{H, G}<\infty$, so that $\|\Psi\|_{B, G}<\infty$, and we conclude that $A_{q, \mu}(G) \subset B_{\alpha, \mu}(G)$.

Suppose next that $q \leqq 1$ and $\Psi \varepsilon A_{\alpha, \mu}(G)$. Part (b) of Lemma 1 implies the existence of $F$ holomorphic in $U$ such that $\Psi / F=f \varepsilon A_{1}$. But, as Metzger and Rao point out, $A_{1}=\{0\}$, so that $f \equiv 0$, and finally $\Psi \equiv 0$. It follows that $A_{a, \mu}(G)=\{0\}$ for $q \leqq 1$. The proof is complete.

4. Proof of Lemma 1. Since $G$ is finitely generated, the closure of the fundamental domain $R$ contains at most finitely many elliptic fixed points $\eta_{1}, \cdots, \eta_{\text {。 }}$ and finitely many parabolic fixed points $\pi_{1}, \cdots, \pi_{r}$. As is shown in $[5, \mathrm{pp} .613-$ 614] an automorphic form which is analytic at the point $\eta_{i}$ has a zero there of order at least $a_{i}$, where $a_{i}$ is an integer $\geqq 0$ depending only upon $G, q, \mu$, and $\eta_{i}$. We say that $a_{i}$ is the minimal order of a zero at $\eta_{i}$. Likewise at $\pi_{i}(1 \leqq$ $j \leqq r$ ) an automorphic form $f$ of degree $-2 q$ has an expansion of the form

$$
f(z)=\left(z-\pi_{j}\right)^{-2 q} \sum_{n=-\infty}^{\infty} c_{n} t^{n+\kappa_{i}}
$$

where $0 \leqq \kappa_{i}<1, t=\exp \left(2 \pi i A_{j} z / \lambda_{i}\right)$. Here $\lambda_{j}>0$ and $A_{j}$ is a linear fractional transformation such that $A_{j}(U)=$ upper half-plane and $A_{j}\left(\pi_{i}\right)=i \infty$. If $f$ is holomorphic in $U$, then the expansion is valid in all of $U$; if $f \varepsilon A_{a, \mu}(G)$, the expansion assumes the form

$$
f(z)=\left(z-\pi_{i}\right)^{-2 a} \sum_{n=0}^{\infty} c_{n} t^{n+\kappa_{j}} .
$$


If $n_{0}$ is the least value of $n$ such that $c_{n} \neq 0$ in (9), then we say that $f$ has $a$ zero of order $n_{0}+\kappa_{j}$ at $\pi_{i}$. If $q \geqq 1, f \varepsilon A_{a, \mu}(G)$ has a positive order zero at $\pi_{i}$ for $1 \leqq j \leqq r$; if $q<1$, then $f \varepsilon A_{\alpha, \mu}(G)$ may have a zero of order zero at $\pi_{i}$. (This is possible of course only if $\kappa_{j}=0$.) Thus if $q \geqq 1, f \varepsilon A_{a, \mu}(G)$ has a zero at $\pi_{j}$ of order at least

$$
\kappa_{j}^{\prime}= \begin{cases}1 & \text { if } \quad \kappa_{j}=0 \\ \kappa_{j} & \text { if } \quad \kappa_{j}>0\end{cases}
$$

and if $q<1$ such $f$ has a zero at $\pi_{j}$ of order at least $\kappa_{j}$. We call $\kappa_{i}^{\prime}$ the minimal order of a zero at $\pi_{j}$ if $q \geqq 1$, and $\kappa_{j}$ the minimal order of a zero at $\pi_{i}$ if $q<1$. It should be emphasized that here a "minimal order zero" is the minimum order zero of an element of $A_{\alpha, \mu}(G)$, whereas in [5] the phrase refers to the minimum order zero of an element of $H_{a, \mu}(G)$. At the elliptic points there is no distinction and if $q \geqq 1$ there is no distinction at the parabolic points. There may be a difference at the parabolic points if $q<1$.

In $\$ 5$ we shall introduce the Poincaré series and use them to construct the function $F$ in the following result. (Compare with the Modified Main Lemma of [5].)

Lemma 2. Given a real number $q$ and a multiplier system $\mu$ corresponding to the degree $-2 q$ there exists an automorphic form $F$, of degree $-2 q$ and multiplier system $\mu$, such that

(i) $F$ is holomorphic in $U$;

(ii) $F$ has minimal order zeros at $\eta_{1}, \cdots, \eta_{0}$ and $\pi_{1}, \cdots, \pi_{r}$;

(iii) $F$ is bounded away from zero in every domain of the form

$$
\mathfrak{R}^{*}=R-\bigcup_{i} K_{i}-\bigcup_{i} N_{i}
$$

where $K_{i}$ is an open disc centered at $\eta_{i}$ and $N_{i}$ an open disc centered at $\pi_{i}$. Furthermore if $q \geqq 1, F \varepsilon H_{q, \mu}(G)$.

We show that the function $F$ of Lemma 2 satisfies the conclusion of Lemma 1, provided only that the $K_{i}$ and $N_{i}$ are nonoverlapping, the closure of each $K_{i}$ is contained in $U$, and the closure of $N_{i}$ does not intersect a free side of $R$.

(a) Suppose $q>1$ and $\Psi \varepsilon A_{a, \mu}(G)$.

Let $f=\Psi / F$, with $F$ as in Lemma 2 . We show that $f \varepsilon A_{a}$, that is,

$$
\iint_{U}|f(z)|\left(1-|z|^{2}\right)^{a-2} d x d y<\infty
$$

We have

$$
\iint_{U}=\iint_{G Q}=\iint_{G \Omega^{*}}+\iint_{G\left(Q-Q^{*}\right)},
$$


with the convention

$$
\iint_{G S}=\sum_{T \in G} \iint_{T(S)}, \quad S \subset U
$$

From the transformation properties of $\Psi$ and $F$ it follows that $f=\Psi / F$ is invariant with respect to $G$, that is, $f(T z)=f(z)$ for all $T$ in $G$. Since $\Psi \varepsilon A_{\alpha, \mu}(G)$ it has zeros at $\eta_{1}, \cdots, \eta_{\mathrm{s}}$ and $\pi_{1}, \cdots, \pi_{r}$ of order at least equal to the minimal order at each of these points. It follows that $f$ is holomorphic in $R-R^{*}$ and, in fact, bounded in $R-R^{*}$. By the invariance of $f$ it is bounded in $\bigcup_{T_{\varepsilon} G} T\left(R-\Omega^{*}\right)$ and with the same bound as in $R-R^{*}$. Since $q>1$ we have

$$
\begin{aligned}
\iint_{G\left(Q-Q^{*}\right)} & |f(z)|\left(1-|z|^{2}\right)^{q-2} d x d y \\
\quad \leqq & K \iint_{U}\left(1-|z|^{2}\right)^{q-2} d x d y=K \frac{\pi}{q-1}, \text { with some } K>0 .
\end{aligned}
$$

We know there exists $\epsilon=\epsilon\left(\Omega^{*}\right)>0$ such that $|F(z)| \geqq \epsilon$ for $z \varepsilon R^{*}$. Then

$$
\begin{aligned}
\iint_{\mathbb{Q}^{*}}|f(z)|\left(1-|z|^{2}\right)^{\alpha-2} d x d y & <\epsilon^{-1} \iint_{\mathbb{R}^{*}}|\Psi(z)|\left(1-|z|^{2}\right)^{\alpha-2} d x d y \\
& \leqq \epsilon^{-1} \iint_{\Omega^{\prime}}|\Psi(z)|\left(1-|z|^{2}\right)^{\alpha-2} d x d y<\infty,
\end{aligned}
$$

since $\Psi \varepsilon A_{a, \mu}(G)$. It remains only to show that (13) implies

$$
\iint_{G \Omega^{*}}|f(z)|\left(1-|z|^{2}\right)^{a-2} d x d y<\infty,
$$

since (12) and (14) imply (11). We have

$$
\begin{aligned}
\iint_{G Q^{*}}|f(z)|\left(1-|z|^{2}\right)^{a-2} d x d y= & \sum_{T_{\varepsilon} \in} \iint_{T_{R^{*}}}|f(z)|\left(1-|z|^{2}\right)^{a-2} d x d y \\
& =\sum_{T_{\varepsilon} G} \iint_{\mathcal{Q}^{*}}|f(T \xi)|\left(1-|T \xi|^{2}\right)^{a-2}\left|T^{\prime}(\xi)\right|^{2} d u d v .
\end{aligned}
$$

Since $f(T \xi)=f(\xi)$ and

$$
\left(1-|T \xi|^{2}\right)^{a-2}\left|T^{\prime}(\xi)\right|^{2}=\left(1-|\xi|^{2}\right)^{q-2}\left|T^{\prime}(\xi)\right|^{\alpha},
$$

the integral equals

$$
\begin{array}{rl}
\sum_{T_{\varepsilon} G} \iint_{Q^{*}}|f(\xi)|\left(1-|\xi|^{2}\right)^{a-2}\left|T^{\prime}(\xi)\right|^{a} & d u d v \\
& =\iint_{Q^{*}}|f(\xi)|\left(1-|\xi|^{2}\right)^{a-2}\left\{\sum_{T_{\varepsilon} G}\left|T^{\prime}(\xi)\right|^{a}\right\} d u d v .
\end{array}
$$

If we write (as is usual)

$$
T(\xi)=(\alpha \xi+\beta) /(\bar{\beta} \xi+\bar{\alpha}), \quad|\alpha|^{2}-|\beta|^{2}=1,
$$


then $\left|T^{\prime}(\xi)\right|^{q}=|\bar{\beta} \xi+\bar{\alpha}|^{-2 a}=|\bar{\beta}|^{-2 a}|\xi+\bar{\alpha} / \bar{\beta}|^{-2 a}$. Since $\{\bar{\alpha} / \bar{\beta} \mid T \varepsilon G\}$ accumulates only at limit points of $G$ it follows that $|\xi+\bar{\alpha} / \bar{\beta}| \geqq \delta>0$ uniformly on $\mathbb{R}^{*}$, and therefore, since $q>1$,

$$
\sum_{T \varepsilon G}\left|T^{\prime}(\xi)\right|^{\alpha} \leqq \delta^{-2 a} \sum_{T \varepsilon G}|\bar{\beta}|^{-2 a}=K, \text { for } \xi \varepsilon Q^{*}
$$

(see $[4$, p. 106]). From this we obtain

$$
\iint_{G \Omega^{*}}|f(z)|\left(1-|z|^{2}\right)^{a-2} d x d y \leqq K \iint_{Q^{*}}|f(\xi)|\left(1-|\xi|^{2}\right)^{a-2} d u d v<\infty .
$$

This completes the proof of Lemma 1(a).

(b) Suppose $q \leqq 1$. Again let $\Psi \varepsilon A_{a, \mu}(G)$ and put $f=\Psi / F$, with $F$ as in Lemma 2. If $q<1 F$ may not be in $H_{a, \mu}(G)$, but this is of no consequence. $f$ is invariant with respect to $G$, holomorphic in $U$, regular at the parabolic points of $R$, and bounded in $R-R^{*}$ (hence in $\bigcup_{T_{\varepsilon} G} T\left(R-R^{*}\right)$ ). We shall show that $f \varepsilon A_{1}$, that is,

$$
\iint_{U}|f(z)|\left(1-|z|^{2}\right)^{-1} d x d y<\infty
$$

First consider

$$
\begin{aligned}
\iint_{G G^{*}}|f(z)|\left(1-|z|^{2}\right)^{-1} d x d y & =\sum_{T_{\varepsilon} G} \iint_{T_{G^{*}}}|f(z)|\left(1-|z|^{2}\right)^{-1} d x d y \\
& =\sum_{T_{\varepsilon} G} \iint_{\Omega^{*}}|f(T \xi)|\left(1-|T \xi|^{2}\right)^{-1}\left|T^{\prime}(\xi)\right|^{2} d u d v \\
& =\sum_{T_{\varepsilon} G} \iint_{\Omega^{*}}|f(\xi)|\left(1-|\xi|^{2}\right)^{-1}\left|T^{\prime}(\xi)\right| d u d v
\end{aligned}
$$

since $\left(1-|T \xi|^{2}\right)^{-1}=\left(1-|\xi|^{2}\right)^{-1}\left|T^{\prime}(\xi)\right|^{-1}$. The integral equals

$$
\iint_{\mathcal{Q}^{*}}|f(\xi)|\left(1-|\xi|^{2}\right)^{-1}\left\{\sum_{T_{\varepsilon} G}\left|T^{\prime}(\xi)\right|\right\} d u d v \leqq K \iint_{\mathcal{G}^{*}}|f(\xi)|\left(1-|\xi|^{2}\right)^{-1} d u d v,
$$

as in the proof of Lemma 1 (a). As before, observe that $|F(\xi)| \geqq \epsilon>0$ on $\mathbb{R}^{*}$, so that

$$
|f(\xi)|=|\Psi(\xi)| /|F(\xi)| \leqq \epsilon^{-1}|\Psi(\xi)|, \quad \text { on } \quad a^{*}
$$

Therefore,

$$
\begin{array}{r}
\iint_{G \Omega^{*}}|f(z)|\left(1-|z|^{2}\right)^{-1} d x d y \leqq K \epsilon^{-1} \iint_{\mathscr{Q}^{*}}|\Psi(\xi)|\left(1-|\xi|^{2}\right)^{-1} d u d v \\
\leqq K \epsilon^{-1} \iint_{\mathbb{Q}^{*}}|\Psi(\xi)|\left(1-|\xi|^{2}\right)^{a-2} d u d v
\end{array}
$$

because $q \leqq 1$ (hence $\left(1-|\xi|^{2}\right)^{q-1} \geqq 1$ ). Since $\Psi \varepsilon A_{\alpha, \mu}(G)$, the right hand side of (16) is finite. 
It remains to show that

$$
\iint_{G\left(\Omega-\Omega^{*}\right)}|f(z)|\left(1-|z|^{2}\right)^{-1} d x d y<\infty .
$$

The left hand side of (17) is equal to

$$
\iint_{\mathcal{R}_{-\Omega^{*}}}|f(\xi)|\left(1-|\xi|^{2}\right)^{-1}\left\{\sum_{T_{\varepsilon} G}\left|T^{\prime}(\xi)\right|\right\} d u d v,
$$

as before. Since $|f(\xi)| \leqq K$ on $R-\Omega^{*}$, the integral is

$$
\leqq K \iint_{\mathcal{G}_{-G^{*}}}\left(1-|\xi|^{2}\right)^{-1}\left\{\sum_{T_{\mathfrak{R} G}}\left|T^{\prime}(\xi)\right|\right\} d u d v \text {. }
$$

We now apply Theorem 3 of [9] (see also Lemma 1.5 of [6]), by virtue of which there exists $K^{\prime}>0$ such that

$$
\sum_{T_{\varepsilon} G}\left|T^{\prime}(\xi)\right| \leqq K^{\prime}\left(1-|\xi|^{2}\right)^{-1},
$$

for all $\xi \varepsilon U$. In particular, we have

$$
\begin{aligned}
& \iint_{\mathcal{R}_{-\Omega^{*}}}\left(1-|\xi|^{2}\right)^{-1}\left\{\sum_{T \varepsilon G}\left|T^{\prime}(\xi)\right|\right\} d u d v \\
& \leqq K^{\prime} \iint_{\Omega_{-} \Omega^{*}}\left(1-|\xi|^{2}\right)^{-2} d u d v=K^{\prime} \cdot\left(\text { hyperbolic area of } \mathcal{R}-\mathfrak{Q}^{*}\right)<\infty \text {. }
\end{aligned}
$$

Thus (17) follows, (15) holds, and $f \varepsilon A_{1}$. The proof of Lemma 1 is complete.

5. Proof of Lemma 2. We shall construct $F$ by means of the well-known Poincaré series whose definition follows.

Suppose $h$ is a function, defined and bounded on $U, q$ is a real number $\geqq 1$, and $\mu$ is a multiplier system for the group $G$ and the degree $-2 q$. Then the Poincaré series $\theta_{a, G}^{\mu}(h)=\theta(h)$ is defined for $z$ in $U$ by

$$
\theta_{a, G}^{\mu}(h)=\sum_{\boldsymbol{v}, G} h(V z) \bar{\mu}(V) V^{\prime}(z)^{a} .
$$

Since $G$ is of the second kind and $q \geqq 1$ the series (18) converges absolutely at each point of $U$ and uniformly on compact subsets of $U$ (see [5, p. 605]). In particular if $h \varepsilon H^{\infty}(U)$, then $\theta(h)$ is holomorphic in $U$. Furthermore, the absolute convergence and the consistency condition (5) imply that $\theta(h)$ satisfies (4). Indeed, in [5, Proposition 3] it is shown that $\theta(h) \varepsilon H_{a, \mu}(G)$, provided $h \varepsilon H^{\infty}(U)$ and $q \geqq 1$.

First suppose $q \geqq 1$. With $\mu$ a corresponding multiplier system, let $F_{0}=$ $\theta_{a, G}^{\mu}(1) \varepsilon H_{a, \mu}(G)$. The proof of Proposition 3 of [5] actually proves somewhat more, namely, that the series $\theta(1)$ converges uniformly on compact subsets of (and therefore that $F_{0}$ is holomorphic in) a region of the form

$$
\Omega_{0}=\varsigma-L-X=\Omega-X \text {. }
$$


Here $S$ is the Riemann sphere, $L$ is the set of limit points of $G$ in $\mathcal{S}$, and $X$ is a countable set of closed half-lines in $\delta$, one joining each point of the form $V^{-1}(\infty)$, $V \varepsilon G$, to $\infty .^{2}$ The half-lines in $X$ can be chosen pairwise disjoint, so that $s-X$ is connected and simply connected. $\Omega=\mathcal{S}-L$ is the set of ordinary points of $G$.

As in [3] and [5], we observe that there is a Jordan region $\hat{U} \supset U$ such that $\hat{U}$ is invariant under $G$ and $\hat{U} \supset \overline{\mathcal{Q}}-\left\{\pi_{1}, \cdots, \pi_{r}\right\}$, where $\overline{\bar{Q}}$ is the closure of $R$ in the ordinary topology of $s$. Furthermore $\hat{\theta}$ can be so chosen that $\hat{\theta} \subset \Omega_{0}$ (i.e., $\hat{U}$ is disjoint from $X$ and $L$ ) and $\hat{U} / G$ is a compact bordered Riemann surface, with finitely many punctures (in fact, $r$ punctures-one corresponding to each parabolic point $\pi_{i}$ ). Indeed, $\hat{U}$ can be constructed by first adding to $R$ a suitably small "cap" at each of the finitely many free sides of $\mathcal{R}$, so that the resultant set, $\widehat{R}$, contains the free sides of $R$, excluding the endpoints. $\hat{U}$ is then defined to be $\bigcup_{T \varepsilon G G} T(\widehat{\mathbb{R}}) .^{3}$ It follows that $\widehat{\mathbb{R}}$ is a fundamental domain for $G$ in $\hat{U}$. Let $U^{+}$be the union of $U$ and the parabolic points of $G$ (these are all on $\partial U$ ) ; let $\hat{U}^{+}$be the union of $\hat{U}$ and the parabolic points of $G$. Then

$$
\begin{aligned}
& U^{+}=\bigcup_{r_{\varepsilon} G} T\left(R \cup\left\{\pi_{1}, \cdots, \pi_{r}\right\}\right), \\
& \hat{U}^{+}=\bigcup_{r_{\varepsilon} G} T\left(\hat{\Omega} \cup\left\{\pi_{1}, \cdots, \pi_{r}\right\}\right) .
\end{aligned}
$$

(Note that $\hat{\Omega}-Q$ consists of ordinary points of $G$ only; since the $\pi_{i}$ are limit points of $G, \pi_{j} \notin \widehat{Q}$.) Define the compact bordered Riemann surfaces $S=U^{+} / G$ and $\hat{S}=\hat{U}^{+} / G$; $\hat{S}$ contains the closure of $S$.

The automorphic form $F_{0}=\theta_{\alpha, G}^{\mu}(1)=\theta(1) \varepsilon H_{\alpha, \mu}(G), q \geqq 1$, is analytic in $\Omega_{0}=\Omega-X$; furthermore $F_{0}$ has singularities at the points $V^{-1}(\infty) \varepsilon \partial \Omega_{0}$ if $G$ contains no rotations about 0 , that is, if $0 \&\left\{\eta_{1}, \cdots, \eta_{e}\right\}$. Thus we temporarily assume that 0 is not an elliptic fixed point of $G$ and conclude that $F_{0}$ is not identically 0 . (If $0 \varepsilon\left\{\eta_{1}, \cdots, \eta_{e}\right\}, F_{0}$ may or may not be $\equiv 0$, depending upon $\mu$ and q.) Since $\hat{U} \subset \Omega_{0}, F_{0}$ is analytic in $\hat{U} . \hat{S}$ is an open Riemann surface; thus the Mittag-Leffler theorem holds for $\hat{S}$ and we may conclude that there exists a function $h$ meromorphic in $\hat{U}$ with poles suitably chosen to yield the following properties:

(a) $h(V z)=h(z)$ for all $V \varepsilon G, z \varepsilon \hat{U}$;

(b) $F=h \cdot \theta(1)=\theta(h)$ is analytic in $\hat{U}$ and zero-free in $R-\left\{\eta_{1}, \cdots, \eta_{0}\right\}$;

(c) $F$ has minimal order zeros at $\eta_{1}, \cdots, \eta_{0}$ and $\pi_{1}, \cdots, \pi_{r}$.

(This $h$ is obtained as a meromorphic function on $\hat{S}$ and then interpreted on the uniformizing domain $\hat{O}^{+}$. Then (a) follows directly.) Clearly, $F$ satisfies conditions (i) and (ii) of Lemma 2 and the functional equations (4). Thus $F$ is zero-free in $\hat{U}-\bigcup_{T_{\varepsilon} G} T\left\{\eta_{1}, \cdots, \eta_{\varepsilon}\right\}$; since $\hat{U}$ contains $\overline{\mathcal{A}}-\left\{\pi_{1}, \cdots, \pi_{r}\right\}$, $\overline{\mathscr{R}}-\bigcup_{i} K_{i}-\bigcup_{i} N_{i}$ is a compact subset of $\hat{U}-\bigcup_{T_{2} G} T\left\{\eta_{1}, \cdots, \eta_{\bullet}\right\}$. Hence $F$ is bounded away from zero in $\overline{\mathcal{A}}-\bigcup_{i} K_{i}-\bigcup_{i} N_{i} \supset \mathcal{R}^{*}$, so that condition (iii) of Lemma 2 holds for $F$. $F$ is holomorphic in $\widehat{U}$ and $\bar{R}-\bigcup_{i} N_{i}$ is a compact subset of $\hat{U}$; thus $F$ is bounded on $\bar{R}-\bigcup_{i} N_{i}$. But $F$ is also bounded in each $N_{i}$ since, with $q \geqq 1, F$ has a positive order zero at each $\pi_{i}$. Thus $F \varepsilon H_{q, \mu}(G)$ 
and the proof of Lemma 2 is complete for $q \geqq 1$, under the assumption that no $\eta_{i}=0$.

Still under this latter assumption, suppose $q<1$. In this case put

$$
F_{0}=\theta_{2, G}^{I}(1) / \theta_{2-\alpha, G}^{\bar{\mu}}(1),
$$

a nontrivial automorphic form of degree $-2 q$ and multiplier system $\mu$. Here $I$ is the identically 1 multiplier system on $G$. Note that $2-q>1$ and that $\bar{\mu}$ is a multiplier system for the degree $4-2 q$. Therefore the definition (19) is legitimate. Since the denominator of (19) may have zeros in $\hat{\theta}$ we conclude only that $F_{0}$ is meromorphic in $\hat{U}$. But since $\hat{S}$ is open there exists a function $h$, meromorphic in $\hat{U}$ and invariant with respect to $G$, whose zeros and poles are chosen so that:

(a) $F=h \cdot F_{0}$ is analytic in $\widehat{O}$ and zero-free in $\widehat{Q}-\left\{\eta_{1}, \cdots, \eta_{e}\right\}$;

(b) $F$ has minimal order zeros at $\eta_{1}, \cdots, \eta_{e}$ and $\pi_{1}, \cdots, \pi_{r}$.

As before it follows that $F$ is an automorphic form of degree $-2 q$ and multiplier system $\mu$ satisfying conditions (i), (ii), and (iii) of Lemma 2.

It remains to remove the restriction that no $\eta_{i}=0$. Suppose $\eta_{1}=0$. Let $z_{0}$ be any point in $U$ which is not an elliptic fixed point of $G$; that is,

$$
z_{0} \notin \bigcup_{T \mathfrak{Q} G} T\left\{\eta_{1}, \cdots, \eta_{e}\right\} \text {. }
$$

(This is possible since $G$ is countable.) Let $A$ be any linear fractional transformation fixing $U$, such that $A^{-1}(0)=z_{0}$. Put $G^{\prime}=A G A^{-1}$; then 0 is not an elliptic fixed point of $G^{\prime}$ and $G^{\prime}$ is once again a finitely generated Fuchsian group of the second kind acting on $U$. As is observed in [5, p. 602], a function $F$ satisfies (4) with respect to $G^{\prime}$, the degree $-2 q$, and the multiplier system $\mu^{*}$ if and only if $F_{A}=(F \circ A) \cdot\left(A^{\prime}\right)^{a}$ satisfies (4) with respect to $G$, the degree $-2 q$, and the multiplier system $\mu$. Here $\mu^{*}$ is a specific multiplier system for $G^{\prime}$ which can be calculated in terms of $\mu, A$, and $q ; A^{\prime}$ is the derivative of $A$. In particular, Lemma 1 of [5] shows that $F_{A} \varepsilon H_{q, \mu}(G)$ if and only if $F \varepsilon H_{\alpha, \mu^{*}}\left(G^{\prime}\right)$. Since 0 is not an elliptic fixed point of $G^{\prime}$, there exists $F$ satisfying the conclusions of Lemma 2 for the group $G^{\prime}$ and the multiplier system $\mu^{*}$. Then $F_{A}$ satisfies these conclusions for the group $G$ and the multiplier system $\mu$. The proof of Lemma 2 is complete.

6. Concluding remarks. All of these results can be carried over to the more general situation in which $U$ is replaced by a region $D$, conformally equivalent to $U$. The corresponding Banach spaces are denoted $A_{\alpha, \mu}(D, G), B_{\alpha, \mu}(D, G)$, and $H_{a, \mu}(D, G)$. (See [5] for the definitions.) I would like to take this opportunity to observe that the map $f \rightarrow \theta_{q, G}^{\mu}(f)$ is a continuous map of $H_{q}(D)$ into $H_{\alpha, \mu}(D, G)$. In Proposition 3 of [5] it is proved only that this map is into; T. A. Metzger has observed that the continuity follows from the Closed Graph Theorem.

The method of proof of Lemma 2 can be used to show that $H_{\alpha, \mu}(G) \neq\{0\}$ for all real $q$, with a corresponding multiplier system $\mu$. Thus $B_{q, \mu}(G) \neq 0$ 
for $q \geqq 0$ and $A_{\alpha, \mu} \neq\{0\}$ for $q>1$. In Lemma 1 , note that if $\Psi \varepsilon H_{\alpha, \mu}(G)$ and $q \geqq 1$, then $\Psi / F \& H_{0, i}(G) \subset H^{\infty}$.

\section{FOOTNOTES}

1. Bers claims the result only for integral $q \geq 2$; his proof, however, is valid for all real $q$.

2. In $[5$, p. 610] it is erroneously asserted that $\theta(1)$ is holomorphic in $\Omega$. The error has no effect on the results of [5], however.

3. For a detailed construction of $\hat{U}$ and a complete proof of its properties, see [10].

\section{REFERENCES}

1. L. Bers, Automorphic forms and Poincaré series for infinitely generated Fuchsian groups, Amer. J. Math. 87 (1965), 196-214.

2. D. Drasin \& C. J. EarLe, On the boundedness of automorphic forms, Proc. Amer. Math. Soc. 19 (1968), 1039-1042.

3. C. J. Earle \& A. Marden, Projections to automorphic functions, Proc. Amer. Math. Soc. 19 (1968), 274-278.

4. L. R. Fond, Automorphic functions, New York, McGraw-Hill, 1929; 2nd ed. New York Chelsea, 1951.

5. M. I. KNopP, A corona theorem for automorphic forms and related results, Amer. J. Math. 91 (1969), 599-618.

6. T. A. MetzGer, Doctoral Dissertation, Purdue University.

7. T. A. Metzger \& K. V. R. Rao, On integrable and bounded automorphic forms, Proc. Amer. Math. Soc. 28 (1971), 562-566.

8. - On integrable and bounded automorphic forms, II, (to appear).

9. K. V. R. RAO, Fuchsian groups of convergence type and Poincaré series of dimension -2, J. Math. Mech. 18 (1969), 629-644.

10. M. Sheingorn, Doctoral Dissertation, University of Wisconsin, 1970.

University of Illinois, Chicago

Date communicated: JULY 6, 1972 